\title{
Small Cell Neuroendocrine Carcinoma of Tonsil - A Rare Occurrence
}

\author{
${ }^{1}$ Peoli Mukutawat, ${ }^{2}$ Bibhavendra Kumar Singh, ${ }^{3}$ Kunwar Prativyom \\ Senior girls hostel, PGIMS, Rohtak
}

\begin{abstract}
\section{Introduction:}

Small cell neuroendocrine carcinomas of tonsil are extremely rare cancers and only a few cases have been reported till date. They carry a poor prognosis. Paraneoplastic syndromes like SIADH, Cushing's syndrome and Eaton-Lambert myasthenic syndrome can be found associated with these carcinomas. The tumor can metastasize to liver, lungs, bone, brain and skin.
\end{abstract}

\section{$>$ Case Presentation:}

A 70-year-old male presented with a 2-month history of pain in throat which was moderate to severe in intensity and intermittent. Local examination of oropharynx revealed a $5 \times 5 \mathrm{~cm}$ ulcero-proliferative growth over the right tonsil extending to right side of base of tongue, vallecula, and right retromolar trigone. Multiple matted lymph nodes were palpable in the right cervical region. Histopathological examination of tissue sample from the growth over the right tonsil revealed small cell neuroendocrine carcinoma. On immunohistochemistry, chromogranin and CD56 were positive. Patient received 2-courses of neoadjuvant chemotherapy with cisplatin and etoposide. Then the patient was planned for external beam radiation therapy with dose of $60 \mathrm{~Gy}$ in 30 fractions in 6 weeks.

\section{$>$ Discussion:}

Small cell neuroendocrine carcinoma of tonsil are likely to be very aggressive with a tendency of developing early regional lymphatic and systemic metastases. More research and clinical trials shall be explored to obtain a standard treatment strategy for these patients.

\section{INTRODUCTION}

Neuroendocrine carcinoma (NEC) is a malignant epithelial cancer with neuroendocrinal morphology. Small cell neuroendocrine carcinomas are poorly differentiated neuroendocrine tumors. They commonly originate in pulmonary, gastrointestinal tract and genitourinary system. They have also been reported to occur in many other organs throughout the body. ${ }^{1}$ The incidence of small cell NEC is low in head and neck region. In head and neck region, larynx is relatively most commonly involved site, followed by salivary glands, nasal cavity and paranasal sinuses. ${ }^{2}$ Small cell neuroendocrine carcinoma (NEC) that originates in the tonsil is extremely rare and only a few cases have been reported since it was first seen by Koss et al. in 1972 .
Small cell neuroendocrine carcinoma of tonsil carries a poor prognosis. ${ }^{3}$

Neuroendocrine carcinoma is positive for choromogranin A, synaptophysin and CD56 endocrine markers. A Ki67 or mitotic index of $20 \%$ or more is also necessary for diagnosing NEC. Tumors with less than $20 \%$ Ki67 positivity are diagnosed as neuroendocrine tumors. ${ }^{4}$

As of now, the treatment strategy for small cell neuroendocrine carcinoma has not been properly formulated due to the sparsity of data. Here we present a rare case of primary small cell neuroendocrine carcinoma arising from the tonsil.

\section{CASE PRESENTATION}

A 70-year-old male presented with a 2-month history of pain in throat which was gradual in onset, progressive, moderate to severe in intensity, intermittent, and with no aggravating or relieving factors. The patient was a chronic smoker since 40-years. There was no history of any chronic illness or history of cancer in the family and the patient was not receiving any medication.

General physical examination and systemic examination were normal. Local examination of oropharynx revealed a $5 \times 5 \mathrm{~cm}$ ulcero-proliferative growth over the right tonsil extending to right side of base of tongue, vallecula, and right retromolar trigone. Multiple matted lymph nodes were palpable in the right cervical region involving levels IB, II, III, and IV; largest lymph node measuring $7 \times 5 \mathrm{~cm}$. Hematological and biochemical profile were within normal limits. Chest X-ray was also normal.

CECT scan of face and neck revealed $5 \times 4.5 \mathrm{~cm}$ heterogeneously enhancing lobulated soft tissue mass lesion over the right tonsil with obliteration of right tonsillo-lingual sulcus and extending to right side of base of tongue and thickening of right lateral pharyngeal wall superiorly upto soft palate. Multiple enlarged lymph nodes were noted in the right side of neck, largest being $6.5 \times 5$ $\mathrm{cm}$.

A biopsy was taken from the growth over the right tonsil and the histopathological examination revealed small cell neuroendocrine carcinoma. On immunohistochemistry, chromogranin and CD56 were positive. 
Patient received 2-courses of neoadjuvant chemotherapy with injection cisplatin $150 \mathrm{mg}$ and injection etoposide $150 \mathrm{mg}$, intravenously. Thereafter the disease had progressed in size and there was no response. Then the patient was planned for external beam radiation therapy with dose of $60 \mathrm{~Gy}$ in 30 fractions in 6 weeks.

\section{DISCUSSION}

Small cell neuroendocrine carcinomas of tonsil are extremely rare cancers and only a few cases have been reported since it was first seen by Koss et al. in 1972. They carry a poor prognosis. ${ }^{3}$ They most commonly occur during 5 th to 7 th decade of life, and are more common in males than in females (male : female ratio $=2: 1$ ). The patient usually presents with a painless neck mass. Paraneoplastic syndromes like SIADH, Cushing's syndrome and EatonLambert myasthenic syndrome can be found associated with these carcinomas. ${ }^{2}$ The tumor can metastasize to liver, lungs, bone, brain and skin. ${ }^{5}$

The treatment options for small cell neuroendocrine carcinoma of tonsil are chemotherapy, radiotherapy, surgical resection or a combinations of these modalities. Though, as of now, the treatment strategy for small cell neuroendocrine carcinoma has not been properly formulated due to the sparsity of data.

Small cell neuroendocrine carcinoma of tonsil are likely to be very aggressive with a tendency of developing early regional lymphatic and systemic metastases. Due to this reason, it may be considered as a systemic disease. ${ }^{2}$ More research and clinical trials shall be explored to obtain a standard treatment strategy for these patients.

\section{REFERENCES}

[1]. Jang H, Yuk SM, Kim JO, Han DS. A rare case of primary malignant small cell carcinoma combined with urothelial cell carcinoma in the ureter. World $\mathbf{J}$ Surg Oncol. 2013;11:181.

[2]. Renner G. Small cell carcinoma of the head and neck: a review. Semin Oncol. 2007;34:3-14.

[3]. Koss LG, Spiro RH, Hajdu S. Small cell (oat cell) carcinoma of minor salivary gland origin. Cancer. 1972;30:737-741.

[4]. Hou X, Wei JC, Wu JX, Wang X, Fu JH, Lin P, et al. Multidisciplinary modalities achieve encouraging long-term survival in resectable limited-disease esophageal small cell carcinoma. PLoS One. 2013;8(7):e69259 Epub 2013/07/23.

[5]. Abedi E, Sismanis A: Extrapulmonary oat-cell carcinoma of the tonsil. Ear Nose Throat J 1987;66:112- 115 . 